\title{
Family Relationships and Suicide Ideation: The Mediating Roles of Anxiety, Hopelessness, and Depression in Adolescents
}

\author{
Paria Ahookhosh, ${ }^{1}$ Bahman Bahmani, ${ }^{1}$ Ali Asgari, ${ }^{2}$ and Hossein Hassanian Moghaddam ${ }^{3, *}$ \\ ${ }^{1}$ Department of Counselling, University of Social Welfare and Rehabilitation Sciences, Tehran, IR Iran \\ ${ }^{2}$ Department of Psychology, Kharazmi University, Tehran, IR Iran \\ ${ }^{3}$ Department of Clinical Toxiology, Loghman-Hakim Hospital, Shahid Beheshti University of Medical Sciences, Tehran, IR Iran \\ "Corresponding author: Hossein Hassanian Moghaddam, Department of Clinical Toxiology, Loghman-Hakim Hospital, Shahid Beheshti University of Medical Sciences, Tehran, \\ IR Iran. Tel: +98-2155419534, E-mail: hassanian@sbmu.ac.ir
}

Received 2015 July 15; Revised 2015 November 13; Accepted 2015 December 05.

\begin{abstract}
Background: Several studies have shown that the suicide trend in Iran is rising. Although there is a growing number of studies that identify a number of the risk and protective factors associated with suicidal behaviors in adolescents, only a few studies have integrated this knowledge for the purpose of validating more complex models of adolescent suicidal behaviors in the Middle East. objectives: The purpose of this study was to explore a theoretically derived model positing that anxiety, depression, and hopelessness mediate between family relationships as indicators of burdensomeness and belongingness in interpersonal-psychological theory and suicide ideation among adolescent suicidal attempters.

Patients and Methods: This study develops and tests a model of suicide ideation with family relationships as antecedents, and latent variables as depression, hopelessness, and anxiety. In particular, we investigated 120 consecutive adolescent suicidal attempters who were assessed in terms of their Family Environment Scale (FES) and Beck Depression Inventory (BDI-II), Beck Hopelessness Scale (BHS), Beck Anxiety Inventory (BAI), and Beck Suicide Ideation Scale (BSSI). The final structural models have shown to be fitted to the considered data.

Results: The results showed that family cohesion and expressiveness were stronger predictors of suicide ideation than family conflict. Indeed, family cohesion exerted direct and indirect effects through depression, hopelessness, and anxiety on suicide ideation, and family expressiveness was also directly related to suicide ideation. It was shown that latent variables (depression, hopelessness, and anxiety) were positively correlated with suicide ideation.

Conclusions: The results of the study imply that family cohesion and family expressiveness act as predictive factors against depression, hopelessness, anxiety, and suicide ideation.
\end{abstract}

Keywords: Anxiety, Dysthymic Disorder, Depression, Family Conflict, Suicide, Attempted

\section{Background}

Several studies have shown that the suicide trend in Iran is rising $(1,2)$. Although there have been a growing number of studies that identify a number of the risk and protective factors associated with suicidal behavior in Iran, few of them have integrated this knowledge for the purpose of validating more complex models of adolescent suicidal behaviors $(3,4)$. In particular, studying suicidal behavior in Iran remains critical for more future studies.

In general, it is believed that suicidal behaviors consist of suicide ideation, suicide attempts, and completed suicide (5). Moreover, we know that suicide ideation generally precedes more serious suicidal behaviors, such as attempted and completed suicides (6). This fact motivates us specifically to focus on suicide ideation in this study.

\section{Objectives}

The purpose of this study was to explore a theoretically derived model positing that anxiety, depression, and hopelessness mediate between family relationships as indicators of burdensomeness and belongingness in interpersonal-psychological theory and suicide ideation among adolescent suicidal attempters (7). Figure 1 illustrates the theoretical framework and describes our hypotheses.

\section{Patients and Methods}

\subsection{Participants}

Study participants (Table 1) included 120 (90 females, 30 males) adolescent suicidal attempters hospitalized for deliberate self-poisoning at Loghman-Hakim hospital, Tehran. Their age range was 14-18 years, with a mean age of $16.71(S D=1.35)$ years: $5.7 \%, 14 ; 6.2 \%, 15 ; 10 \%, 16 ; 12.4 \%, 17$; and 


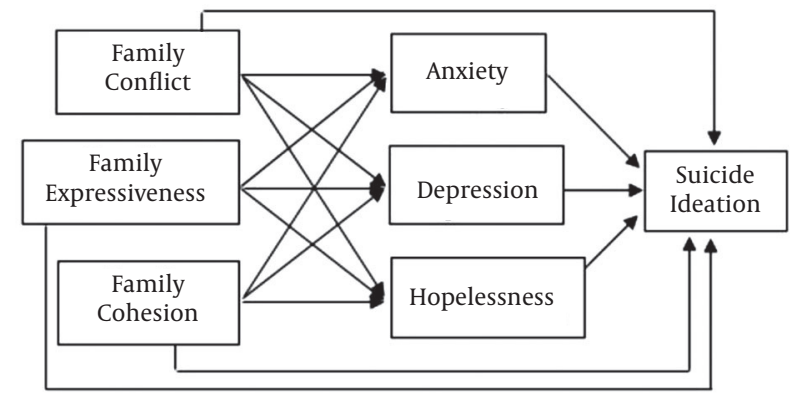

Figure 1. Hypothesized Antecedent Effects Family Cohesion, Expressiveness and Conflict and Mediating Effects Of Anxiety, Depression, and Hopelessness on Adolescent Suicide Risk Behaviors

$23 \%, 18$ years old. Table 1 shows the demographic characteristics of the study population.

\subsection{Procedure}

This prospective observational study involved an analysis of data drawn from the patients who had attempted suicide. The inclusion criteria for participants were: (a) patients who were hospitalized for non-fatal, deliberate selfpoisoning; (b) suicide attempters between the ages of 12 and 18. Exclusion criteria were defined as: (a) poisoning, along with substance abuse, alcohol use, or other illicit drugs; (b) adolescent suicidal attempters who were psychotic; (c) treatment received only in an emergency department; (d) incapable of giving informed consent; (e) insufficient Farsi (Iran's language) to read a questionnaire; and (f) posing a potential threat to the interviewer.

The data used in this study were collected prospectively between June and July 2012. An initial screening was conducted to determine whether an individual met the criteria for participation. After identification of 12-18-yearold admitted patients in the medical toxicology wards, their medical records were studied, and suicide attempters were determined. An individual who met the criteria and consented to participate to a survey was subsequently enrolled into the study voluntarily. The participants were assessed individually using self-report scales. The samples were selected non-randomly and consecutively the day after regaining full consciousness and resolving delirium, if any. Participants were given the Beck depression inventory (DBI-II, Beck, 1996), the Beck scale suicide ideation test (SSI, Beck, 1979), the Beck hopelessness scale (BHS, Beck, 1974), Beck anxiety inventory (BAI, Beck, 1988), and the family environment scale-relationships subscale (FES, Moos \& Moos, 1986).

\subsection{Instruments}

Family Environment Scale (FES): Family relationships (cohesion, expressiveness, and conflict) were measured in terms of the 27 items of the family environment scalerelationships subscale. Each item was rated true or false, with the scores in each of the subscale ranging from 0 to 9 (maximum scores). The FES has shown high reliability in Iran, with test-retest correlations in the three subscales of 0.83 in family cohesion, 0.76 in family expressiveness, and 0.71 in family conflict. Alpha coefficients for the family cohesion, conflict, and expressiveness subscales were 0.79 , 0.73 , and 0.69 , respectively. It should be noted that the discriminate validity of FES is well known (8).

\subsubsection{Suicide Ideation}

Beck Suicide Ideation Scale (BSIS) was used to quantify the intensity of participants' current suicidal intent. Each item consisted of three alternative statements graded in intensity from 0 to 2 , and the total scores ranged from 0 to 38.9 The BSSI was found to have acceptable psychometric properties in an Iranian sample; with concurrent validity (0.76) and reliability $(\alpha=0.95)(9)$.

\subsubsection{Depression}

The Beck Depression Inventory-II (BDI-II) is one of the most commonly used measures of depressive symptoms. The BDI-II is a frequently used 21-item self-report measure of depressive symptoms. The scale has a two-week time frame, and scores for each item range from 0 to 3 . Total scores for BDI-II range from 0 to 63, where higher scores represent a higher occurrence of symptoms associated with depression (10). In 2007, Dobson and Mohamadkhani investigated the convergent validity by brief symptom inventory (BSI, Derogatis, 1993). The BDI-II showed a significant association (0.87) and a high internal consistency in $\operatorname{Iran}(\alpha=0.91)(11)$.

\subsubsection{Hopelessness}

The Beck Hopelessness Scale (BHS) is a 20-item selfreport measure designed to assess an individual's level of hopelessness. It asks individuals to indicate whether statements are true or false (12). Scores can range from 0 to 20 , with higher scores indicating a greater degree of hopelessness. Goudarzi (13) demonstrated that BHS has a good concurrent validity (0.57), a test-retest reliability (0.71), and a Cronbach's alpha (0.79).

\subsubsection{Anxiety}

The Beck Anxiety Inventory (BAI) consists of 21 items, each describing a common symptom of anxiety. The respondent is asked to rate how much he or she has been 
Table 1. Demographic Characteristics of all Male and Female Study Participants

\begin{tabular}{|c|c|c|c|}
\hline Demographic Variable & Total Sample & Male & Females \\
\hline No. of participants & 120 & 30 & 90 \\
\hline \multicolumn{4}{|l|}{ Age } \\
\hline M & 16.7 & 17.2 & 16.5 \\
\hline SD & 1.3 & 0.94 & 1.4 \\
\hline \multicolumn{4}{|l|}{ Suicide attempts (\%) } \\
\hline 1 & 81.7 & 90 & 78.9 \\
\hline 2 & 15.8 & 10 & 17.8 \\
\hline 3 & 0.8 & 0 & 1.1 \\
\hline 4 & 1.7 & 0 & 2.2 \\
\hline \multicolumn{4}{|l|}{ Educational Level } \\
\hline High school & 96.7 & 98.9 & 90 \\
\hline Elementary school & 3.3 & 1.1 & 10 \\
\hline
\end{tabular}

bothered by each symptom over the past week on a 4-point scale ranging from 0 (not at all) to 3 (severely). The items are summed to obtain a total score that can range from 0 to 63 (14). Pervious findings based on a validation study in Iran have shown that BAI has high internal consistency $(\alpha=$ $0.92)$ and test-retest reliability $(r=0.83)$, and the scale has demonstrated proper content validity in this study (0.72) (15).

\subsection{Data-Analysis Strategy}

In this study, we were specifically interested in the association between family relationships and suicidal behaviors. More precisely, we wanted to evaluate the strength of the correlation between family relationships and suicide ideation, when the latent variables were studied. The combination of correlation matrices and graphical path diagrams are used and tested in this study to depict the models.

To evaluate the relation between family relationships and suicide ideation, three structure equation models were constructed with suicide ideation, family relationship and anxiety, depression, and hopelessness. SPSS version 16 and LISREL version 8 software were applied to enter and analyze the data.

\section{Results}

\subsection{Bivariate Results}

Bivariate correlation results are presented in Table 2. Suicide ideation was correlated positively to family conflict $(\mathrm{r}=0.21, \mathrm{P}<0.05)$ and family expressiveness $(\mathrm{r}=-0.24, \mathrm{P}$
$<0.01$ ), but was not significantly related to family cohe$\operatorname{sion}(\mathrm{r}=-0.16)$. In addition, the correlation between suicide ideation and moderate variables were significant. Suicide ideation was positively correlated to anxiety $(\mathrm{r}=0.32, \mathrm{P}<$ $0.01)$ and depression $(\mathrm{r}=0.72, \mathrm{P}<0.01)$. Suicide ideation also has shown a high positive correlation with hopelessness $(r=0.51, \mathrm{P}<0.01)$.

Anxiety, depression, and hopelessness showed a significant correlation to family conflict, expressiveness, and cohesion. It is notable that anxiety ( $\mathrm{r}=0.24, \mathrm{P}<0.05)$, depression $(\mathrm{r}=0.26, \mathrm{P}<0.01)$, and hopelessness $(\mathrm{r}=0.21, \mathrm{P}$ $<0.01$ ) indicated significant positive correlations to family conflict. Anxiety was not significantly correlated to family expressiveness $(\mathrm{r}=-0.09)$. Depression $(\mathrm{r}=-0.24, \mathrm{P}<0.01)$ and hopelessness $(\mathrm{r}=-0.20, \mathrm{P}<0.05)$ were correlated to family expressiveness. Anxiety has little negative correlation to family cohesion $(\mathrm{r}=-0.27, \mathrm{P}<0.01)$, and depression and hopelessness have superlative correlations to family cohesion $(r=-0.35, P<0.01)$.

\subsection{Path Analyses Results}

Model results were reported using the standard coefficients; however, both standardized and unstandardized coefficients are illustrated in Figures 2, 3, and 4.

Figure 2 shows that the fit model was significantly appropriate $(\mathrm{CFI}=1, \mathrm{GFI}=0.99, \mathrm{AGFI}=0.98, \mathrm{RMSEA}=0.000)$. The results obtained from the first path model are described in Figure 2 show that family cohesion has a negative relationship with anxiety $(\mathrm{b}=-2.01, \mathrm{P}<0.01)$ and suicide ideation ( $\mathrm{b}=-0.45, \mathrm{P}<0.05$ ). Furthermore, the relationship between family expressiveness and conflict to anxiety were no longer significant in the path model de- 
Table 2. Correlations Between Suicide Ideation, Depression, Anxiety, Family Conflict, Expression, and Cohesion

\begin{tabular}{|c|c|c|c|c|c|c|c|}
\hline & 1 & 2 & 3 & 4 & 5 & 6 & 7 \\
\hline Suicide ideation & - & & & & & & \\
\hline Depression & $0.72^{\mathrm{a}}$ & - & & & & & \\
\hline Hopelessness & $0.51^{\mathrm{a}}$ & $0.61^{\mathrm{a}}$ & - & & & & \\
\hline Anxiety & $0.32^{\mathrm{a}}$ & $0.53^{\mathrm{a}}$ & $0.35^{\mathrm{a}}$ & - & & & \\
\hline Family expressiveness & $-0.24^{\mathrm{a}}$ & $-0.24^{\mathrm{a}}$ & $-0.20^{\mathrm{b}}$ & -0.09 & $0.37^{\mathrm{a}}$ & - & \\
\hline Family conflict & $0.21^{\mathrm{b}}$ & $0.26^{\mathrm{a}}$ & $0.21^{\mathrm{a}}$ & $0.24^{\mathrm{b}}$ & $-0.53^{\mathrm{a}}$ & $-0.22^{\mathrm{b}}$ & - \\
\hline
\end{tabular}

${ }^{\mathrm{a}} \mathrm{P}<0.01$

${ }^{\mathrm{b}} \mathrm{P}<0.05$.

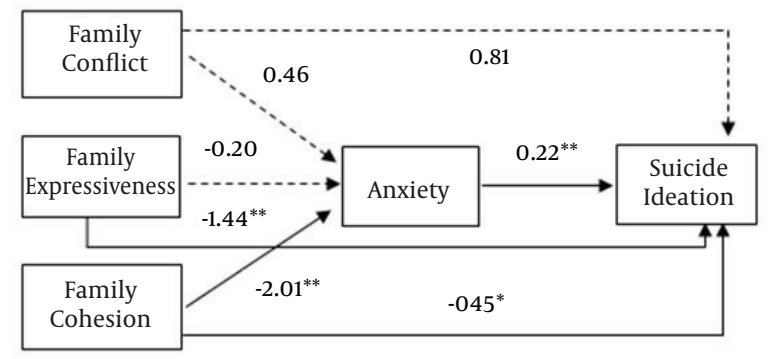

$\mathrm{P}<0.05^{*} \mathrm{P}<0.01^{* *}$

Figure 2. Significant and Insignificant Standardized Coefficients of the Path Model for Anxiety

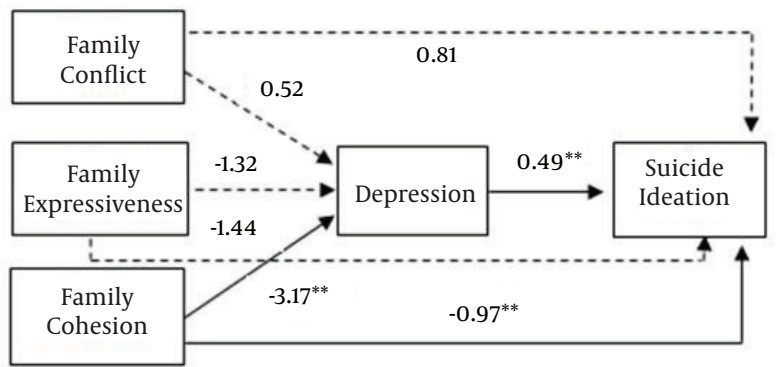

$\mathrm{P}<0 / 05^{*} \quad \mathrm{P}<0 / 01^{* *}$

Figure 3. Significant and Insignificant Standardized Coefficients of the Path Model for Depression

scribed in Figure 2. Family expressiveness has a negative relationship ( $\mathrm{b}=-1.44, \mathrm{P}<0.01$ ), and anxiety has a positive relationship $(\mathrm{b}=0.22, \mathrm{P}<0.01)$ with suicide ideation. Family conflict was not correlated to suicide ideation. We found that there is one indirect path in our model, which was significant (see Table 3). In detail, Table 3 implies that

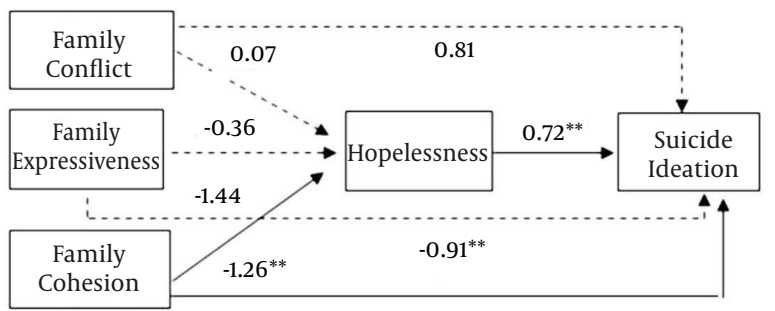

$\mathrm{P}<0.05^{*} \mathrm{P}<0.01^{* *}$

Figure 4. Significant and Insignificant Standardized Coefficients of the Path Mode for Hopelessness

the path from the relationship of family cohesion through anxiety to suicide ideation $(b=-0.45, P<0.01$ ) was significantly negative.

Figure 3 shows that the fit model was significantly appropriate $(\mathrm{CFI}=0.98, \mathrm{GFI}=0.98, \mathrm{AGFI}=0.92, \mathrm{RMSEA}=0.6)$. The results obtained from the second path model and described in Figure 3 show that family cohesion has a negative relationship with depression $(\mathrm{b}=-3.17, \mathrm{P}<0.01)$ and suicide ideation $(b=-0.97, \mathrm{P}<0.01)$. Furthermore, the relationship between family expressiveness and conflict to depression and suicide ideation were no longer significant in the path model described in Figure 3. Depression showed a significant positive relationship with suicide ideation ( $b$ $=0.49, \mathrm{P}<0.01)$ in the hypothesized direction. We found that there is an indirect path in our model, which was significant (see Table 3). In detail, Table 3 implies that the path from family cohesion through adolescent depression to suicide ideation $(b=-1.56, P<0.01)$ was significantly negative.

Figure 4 shows that the fit model was significantly ap- 
Table 3. Tests of the Mediating Roles of Anxiety, Depression, and Hopelessness on Suicidal Behaviors

\begin{tabular}{|c|c|c|c|c|c|c|c|c|}
\hline Model Type & $\chi^{2}$ & df & $\mathbf{P}$ & $\chi^{2} / \mathbf{d f}$ & RMSEA & CFI & FI & AGFI \\
\hline I. Depression & 6.24 & 4 & 0.18 & 1.56 & 0.06 & $0 / 98$ & 0.98 & 0.92 \\
\hline II. Anxiety & 1.55 & 4 & 0.81 & 0.38 & $0 / 000$ & 1 & 99 & 0.98 \\
\hline III. Hopelessness & 4.95 & 3 & 0.26 & $0 / 24$ & $0 / 06$ & 0.99 & $0 / 98$ & 0.92 \\
\hline
\end{tabular}

propriate $(\mathrm{CFI}=0.99, \mathrm{GFI}=0.98, \mathrm{AGFI}=0.92, \mathrm{RMSEA}=0.06)$. The results obtained from the third path model are described in Figure 4 show that family cohesion also has a negative relationship with hopelessness $(\mathrm{b}=-1.26, \mathrm{P}<0.01)$ and suicide ideation $(b=-0.91, P<0.01)$. Furthermore, neither family expressiveness nor family conflict showed a significant relationship to hopelessness and suicide ideation in the path model described in Figure 4. Hopelessness was positively related to suicide ideation $(\mathrm{b}=0.72, \mathrm{P}<0.01)$. We found that there is one indirect path in our model, which was significant (see Table 3). In detail, Table 3 implies that the path from family cohesion through hopelessness to suicide ideation $(b=-0.91, P<0.01)$ is also significantly negative.

\section{Discussion}

The results revealed that family cohesion is consistent with the posited theoretical model and remains a significant predictor of suicide ideation, with or without the contributions of anxiety, hopelessness, and depression. Neither family expressiveness nor family conflict was anticipated via suicide ideation to contribute to feelings of anxiety, hopelessness, and depression. Moreover, family expressiveness was directly related to suicide ideation. Therefore, the results suggest that family cohesion and family expressiveness may be protective factors against suicidal behaviors.

Our results showed that family conflict is not correlated to suicide ideation. Recently, it was believed that family conflict increases when a child reaches adolescence, and hence there is no cause for alarm, as this is a normal stage of development. However, current evidence suggests that conflicts over mundane issues tend to increase during this time, and there is little evidence of a dramatic rise in family conflict from childhood to adolescence (16). A study by Gehring, and colleagues (1990) confirmed that, in the majority of families with adolescents, conflicts are basically between parents and their adolescent and may lead to less cohesion within the family (17). This result is consistent with findings by Street, and colleagues, which indicates the association of family conflict with children's psychosocial adjustment was not easily describable; for example, re- ducing family conflict for African American families may not be sufficient to mitigate psychological adjustment difficulties, because family conflict may not be as directly related (18).

On the other hand, family conflict may have a cultural base. This is something also studied by Park and Kim, who find a relationship between cultural values and communication styles (19). Indeed, they suggested that individual who adhere to the Asian value of emotional self-control may restrain from directly expressing negative emotions toward one another. By responding ambiguously to conflict, or not openly expressing negative feelings, they are able to save the face of those involved in a conflict situation, including him or herself.

Moreover, Park and Kim (2008) (19)showed that collectivistic values positively predicted interpersonal sensitivity and the use of indirect messages. This implies that traditional Asian communication norms reduce the openness and expressiveness used in communication. Family emotional expressiveness as an indicator of family support was related to suicidal behaviors in adolescents and the absence of family expressiveness may contribute to an elevated risk for the onset of suicidal behaviors (20). Conversely, family cohesion may be a protective factor against suicidal behaviors (5). While traditional Asian cultures emphasize group cohesion, the negative consequences of being alienated from valued members of one's social group may be particularly devastating (21).

The results also revealed a pattern of effects that is consistent with other research findings in terms of moderating variables. Indeed, we found that hopelessness was a key predictor of suicidal behaviors. Despite the significant relationship between anxiety and suicidal ideation, it is a very weak relationship. It seems that anxiety does not have a very great direct influence on suicidal behaviors; rather, anxiety may be influenced by suicidal behaviors indirectly, through its strong effects on hopelessness and depression $(20,22)$. Furthermore, depression was significantly related to suicide ideation; however, this association was not considerable, and it was less than the correlation between suicide ideation and hopelessness. It appears that the relationship between depression and suicide ideation is affected by anxiety and hopelessness. 
The present results are in accordance with Beck et al.'s studies, in which hopelessness has been shown to correlate more strongly than depression with suicide intent $(23,24)$. As a result, these findings support the growing number of studies that suggests that hopelessness is more directly related to suicide intent than depression alone.

Overall, our findings are congruent with the notion of belongingness as a protective factor against suicidal behavior. Our findings also do not lend support to the idea that family conflict is one burden on family associated with suicide behaviors. Wimberley, Street et al. came to a similar conclusion as those seen in the study regarding conflict in African American families $(17,25)$. Those proposed that, in some families, the greater absence of conflict may not necessarily lead to more positive outcomes and overall better adjustment of family members; rather, family conflict was perhaps a moderating factor in family cohesion. On the contrary, according to interpersonal-psychological theory, thwarted belongingness and perceived burdensomeness are presumed to be distinct but related constructs. Perceptions of burdensomeness cannot preclude the need to belong (26). Therefore, further research is needed to explore the relationship of conflict with various other family characteristics.

\subsection{Limitations}

One limitation of our study was that cross-sectional data only provided causal inferences between family and psychological variables. It is possible for the variables to have an opposite directionality; for example, individuals who have multiple suicidal attempts (or high levels of suicidal symptoms) may come to view themselves as a burden to others, or adolescents with suicidal ideation are more likely to report having a depressive mood and poor social relationships $(27,28)$. As a result, future studies utilizing a longitudinal design are needed to clarify the casual directions among variables.

It is important to use caution in generalizing the present models, because they were generated from a sample of Iranian adolescents, and so can hardly be generalized to other cultural contexts. Also, due to the focus on clinical samples, the sample size in this study is very small, so it may have led to a reduced generalization of the models.

Finally, it was believed that levels of cohesion lead to different implications for male and female adolescents. For example, Johnson, et al., and Maccoby $(29,30)$ demonstrated that low family cohesion was related to feelings of exclusion, isolation, and loneliness for females. Since this study did not aim to investigate gender differences, our findings must be interpreted cautiously.

\subsection{Conclusion}

Adolescent suicidal behaviors are complex and influenced by the effects of multiple risk factors, especially a lack of family cohesion and expressiveness, and they are mediated by anxiety, hopelessness, and depression. This study provides the first step in the test of a theoretical model for explaining adolescent suicidal behaviors. For mental health professionals, school personnel, and prevention scientists working with at-risk or suicidal adolescents, the findings make salient the need to address multiple co-occurring problem behaviors.

\section{Footnotes}

Authors' Contribution: Study concept and design: Paria Ahookhosh, Bahman Bahmani and Hossein HassanianMoghaddam; analysis and interpretation of data: Ali Asgari, and Bahman Bahmani; drafting of the manuscript: Paria Ahookhosh; critical revision of the manuscript for important intellectual content: Paria Ahookhosh, Bahman Bahmani, Ali Asgari, and Hossein Hassanian-Moghaddam; statistical analysis: Ali Asgari.

Funding/Support: This study was supported by the clinical research development center of Loghman Hakim hospital, Shahid-Beheshti University of Medical Sciences, Tehran, Iran.

\section{References}

1. Kiadaliri AA, Saadat S, Shahnavazi H, Haghparast-Bidgoli H. Overall gender and social inequalities in suicide mortality in Iran, 2006-2010: a time trend province-level study. BMJ Open. 2014;4(8):005227. doi: 10.1136/bmjopen-2014-005227. [PubMed: 25138804].

2. Hassanian-Moghaddam H, Zamani N, Rahimi M, Shadnia S, Pajoumand A, Sarjami S. Acute adult and adolescent poisoning in Tehran, Iran; the epidemiologic trend between 2006 and 2011. Arch Iran Med. 2014;17(8):534-8. [PubMed: 25065275].

3. Moradi A, Akbari Zardkhaneh S, Cheraghi F, Foladvand K. An investigation on the role of family and social factors on suicide of university students. J Family Res. 2009;5(4):487-502.

4. Fadaei Z, Ashouri A, Hooshyari Z, Ezanloo B. Path analysis of locus of control, symptoms of depression and suicidal ideation on academic achievement: the role of gender as moderator.J Fundamentals Mental Health. 2011;13(2):148-59.

5. Joel Wong Y, Uhm SY, Li P. Asian Americans' family cohesion and suicide ideation: moderating and mediating effects. Am J Orthopsychiatry. 2012;82(3):309-18. doi: 10.1111/j.1939-0025.2012.01170.x. [PubMed: 22880969].

6. Fong CL, Shah SA, Maniam T. Predictors of suicidal ideation among depressed inpatients in a Malaysian sample. Suicidology Online. 2012;3:33-41.

7. Joiner TE. Why people die by suicide. Cambridge: Harvard University Press; 2005.

8. Molasharifi S, Vakhshor H, Salimizade MK. Standardize family environment scale Iranian family in Tehran.J Family Res. 2005;1(3):254-68. 
9. Anisi J, FathiAshtiani A, Salimi SH, Ahmadi K. Assessment of the reliability and validity scale Beck's suicidal ideation in soldiers. Military Med. 2004;7(1):33-7.

10. Compton MT, Thompson NJ, Kaslow NJ. Social environment factors associated with suicide attempt among low-income African Americans: the protective role of family relationships and social support. Soc Psychiatry Psychiatr Epidemiol. 2005;40(3):175-85. doi: 10.1007/s00127005-0865-6. [PubMed: 15742221].

11. Dobson KE, Mohammadkhani P. Coordinate psychometric of the Beck depression inventory in a large sample of patients with major depressive disorder [In Persian]. J Rehabil Dis Mental Disord. 2007;8(29):80-6.

12. Smith JM, Alloy LB, Abramson LY. Cognitive vulnerability to depression, rumination, hopelessness, and suicidal ideation: multiple pathways to self-injurious thinking. Suicide Life Threat Behav. 2006;36(4):443-54. doi: 10.1521/suli.2006.36.4.443. [PubMed: 16978098].

13. Goudarzi MA. The study on reliability and validity of Beck hopelessness scale in a group of Shiraz university student. J Social Sci Human Shiraz Uni. 2002;18(2):27-39.

14. Beck TA, Epstein N, Brown G, Steer RA. An inventory for measuring clinical anxiety: psychometric properties. J Consult Clin Psychol. 1988;56(6):893-7. doi: 10.1037/0022-006X.56.6.893.

15. Kaviani H, Mousavi AS. Psychometric characteristics of the Beck anxiety inventory in age and gender classes the Iranian population. $J$ Med Depart, Tehran Uni Med Sci. 2008;66(2):136-40.

16. Olson JR, Goddard HW. Adolescent depression as a function of race and family process. Family Sci Rev. 1996;9(2):123-32.

17. Jackson DD. The predictors of family cohesion and conflict in transracially adoptive families. USA: University of Maryland; 2010.

18. Lee MT, Wong BP, Chow BW, McBride-Chang C. Predictors of suicide ideation and depression in Hong Kong adolescents: perceptions of academic and family climates. Suicide Life Threat Behav. 2006;36(1):8296. [PubMed: 16676629].

19. Park YS, Kim BS. Asian and European American cultural values and communication styles among Asian American and European American college students. Cultur Divers Ethnic Minor Psychol. 2008;14(1):47-
56. doi: 10.1037/1099-9809.14.1.47. [PubMed: 18230000].

20. Thompson EA, Mazza JJ, Herting JR, Randell BP, Eggert LL. The mediating roles of anxiety depression, and hopelessness on adolescent suicidal behaviors. Suicide Life Threat Behav. 2005;35(1):14-34. doi: 10.1521/suli.35.1.14.59266. [PubMed: 15843321].

21. Wong YJ, Koo K, Tran KK, Chiu YC, Mok Y. Asian American college students' suicide ideation: a mixed-methods study. J Couns Psychol. 2011;58(2):197-209. doi: 10.1037/a0023040. [PubMed: 21463030].

22. Meneese WB, Yuterzenka BA. An analysis of adolescent suicidal ideation. Current Psyol. 1992;11(1):51-88. doi:10.1007/BF02686827.

23. Beck AT, Brown G, Berchick RJ, Stewart BL, Steer RA. Relationship between hopelessness and ultimate suicide: a replication with psychiatric outpatients. Am J Psychiatry. 1990;147(2):190-5. doi: 10.1176/ajp.147.2.190. [PubMed: 2278535].

24. Beck AT, Steer RA, Kovacs M, Garrison B. Hopelessness and eventual suicide: a 10-year prospective study of patients hospitalized with suicidal ideation. Am J Psychiatry. 1985;142(5):559-63. doi: 10.1176/ajp.142.5.559. [PubMed: 3985195].

25. Wimberley JC. Family environment and adolescents feelings of hopelessness among low-income, urban African American families. USA: University of Alabama; 2012.

26. Van Orden KA, Lynam ME, Holler D, Joiner TE. Perceived burdensomeness as an indicator of suicidal symptoms. Cogn Ther Res. 2006;30:457-67. doi: 10.1007/s10608-006-9057-2.

27. Van Orden KA, Witte TK, Cukrowicz KC, Braithwaite SR, Selby EA, Joiner TE. The interpersonal theory of suicide. Psychol Rev. 2010;117(2):575600. doi: 10.1037/a0018697. [PubMed: 20438238].

28. Sun RCF, Hui EKP. Psychosocial factors contributing to adolescent suicidal ideation.J Youth Adolescent. 2007;36:775-86. doi:10.1007/s10964006-9139-1.

29. Maccoby EE. Gender and relationships. A developmental account. Am Psychol. 1990;45(4):513-20. doi:10.1037/0003-066X.45.4.513.

30. Johnson HD, LaVoie JC, Mahoney M. Interparental conflict and family cohesion predictors of loneliness, social anxiety, and social avoidance in late adolescence. J Adolesc Res. 2001;16(3):304-18. doi: $10.1177 / 0743558401163004$. 